\title{
ANÁLISIS DE SENDERO PARA ALGUNAS PROPIEDADES DEL FRUTO DE MARACUYÁ (Passiflora edulis f. flavicarpa Deg.)
}

\section{PATH ANALYSIS FOR SOME FRUIT PROPERTIES OF PASSION FRUIT (Passiflora edulis f. flavicarpa Deg.)}

Miguel Espitia C. ${ }^{1}$

Luz E. Vargas O. ${ }^{2}$ Guillermo Martínez F. ${ }^{3}$

\section{RESUMEN}

En Colombia, no se han reportado estudios sobre análisis de sendero para maximizar el progreso por selección en el mejoramiento de maracuyá (Passiflora edulis f. flavicarpa Deg.). El estudio tuvo como objetivos realizar dos análisis de sendero, uno para el peso del fruto (PFR) y otro para el peso de la semilla con arilo (PSC) del fruto de maracuyá, bajo las condiciones que presenta la ciudad de Montería (Córdoba, Colombia). Se utilizaron las correlaciones fenotípicas y genéticas originadas del análisis de 400 frutos, obtenidos de diez lotes comerciales, diez plantas / lote y cuatro frutos / planta (repeticiones), bajo un diseño anidado con frutos dentro de planta y plantas dentro de lotes. El análisis de sendero para PFR señaló que los efectos directos fueron positivos y 2,28 a 16,50 veces, los indirectos, cuando se usaron las correlaciones fenotípicas y 1,93 a 6,90 veces cuando se utilizaron las genéticas. El peso de la cáscara (PCA) fue la variable que mostró el mayor efecto directo sobre el PFR. El análisis para el PSC, al

${ }^{1}$ Ingeniero Agrónomo - Ph.D. Fitomejoramiento, Profesor Titular Universidad de Córdoba, Diagonal 50 No. 8 - 105 - Barrio Villa del Río - Montería (Córdoba). mespitia@sinu.unicordoba.edu.co

${ }^{2}$ Licenciada en Estadística, Profesora Catedrática, Universidad de Córdoba, Montería. luzvargas13@hotmail.com

${ }^{3}$ Licenciado en Matemáticas, M.Sc. Estadística, Profesor TitularUniversidad de Córdoba, Montería. guidomaflo@sinu. unicordoba.edu.co utilizar las correlaciones fenotípicas o genéticas, señaló que en el PFR los efectos directos fueron 3,2 a 80 veces los indirectos. El PFR fue la variable que mostró el mayor efecto directo sobre el peso de la semilla con arilo. Los efectos indirectos vía PFR fueron mayores que los directos en todas las otras variables. El peso del fruto, se puede usar como criterio de selección para la obtención de cultivares de alto rendimiento de PSC del fruto en maracuyá.

Palabras clave: Coeficiente de sendero, peso del fruto, peso semillas con arilo, fitomejoramiento.

\section{SUMMARY}

In Colombia, no studies of path analysis to maximize the selection progress in passion fruit (Passiflora edulis f. flavicarpa Deg.) breeding have been reported. The objectives of this work were, therefore, to estimate two path analysis, one for weight per fruit and the other one for seed weight with aril under environmental conditions of the city of Montería (Cordoba, Colombia). Genetic and phenotypic correlations obtained from the analysis of 400 fruits, collected from ten commercial lots, ten plants/ lot and four fruits/plant (repetitions) were used under a hierarchical design with plants within lot and fruits within plant. Path analysis for weight per fruit showed that the direct effects were positive and 2.28 to 16.50 times, compared to indirect effects, when phenotypic correlations were used and, 1.93 to 6.90 times, when genetic correlations were employed. Peel weight was the variable that caused the largest direct effect on fruit 
weight. Path analysis for seed weight with aril, when the phenotypic or genetic correlations were used, showed that in fruit weight the direct effects were 3.2 to 80 times, compared to indirect effects. Fruit weight was the variable that showed the largest direct effect on seed weight with aril. The indirect effects through fruit weight were higher than the direct effects within the other variables. Fruit weight can be used as selection criteria for obtention of passion fruit cultivars of high pulp yield.

Key words: Path coeficient, fruit weight, weight of seed with aril, plant breeding.

\section{INTRODUCCIÓN}

En Colombia, el cultivo del maracuyá ha sido priorizado, estratégicamente, dentro de la agenda exportadora (Agronet, 2008). El cultivo comercial en el país, se inició en los años 60 y fue en la década de los 80 cuando comenzó su participación en el mercado internacional. La superficie cultivada en 2005 fue de 4.570ha, con una producción de $80,397 \mathrm{t}$, rendimiento promedio de $17,6 t^{h a-1}$ y costos de producción variables, en poblaciones orientadas a mercados, en ciclo de tres años, que oscilan entre US\$180 a 290 por t y ganancias netas de US\$1.500 por hectárea (Schwentesius E Gómez, 2008; Agronet, 2008). Ecuador es el principal exportador de jugo concentrado, superando a Brasil, logrando exportaciones a Estados Unidos, Alemania, Chile, Argentina y Brasil, cuyo uso es reforzar el sabor de otras frutas, sobre todo del durazno, en jugos mezclados, yogur y quesos (Schwentesius \& Gómez, 2008).

La maracuyá es una de las especies hortofrutícolas priorizadas en la agenda interna del departamento de Córdoba, por el valor alimenticio de su jugo y por el potencial que ofrece la utilización de la cáscara y la semilla (Agronet, 2008). Es el tercer productor a nivel nacional, con 887ha, producción de 15.978t y rendimiento de $18 \mathrm{t} \mathrm{ha}{ }^{-1}$. La mayor área, se encuentra localizada en el corregimiento de San Isidro, con rendimientos de 15t ha $^{-1}$, muy por debajo del rendimiento promedio del Ecuador, que es de $25 \mathrm{t} \mathrm{ha}^{-1}$. Esta baja producción es causada entre otros factores por la gran variabilidad de los suelos y manejo agronómico en los campos comerciales, además de la falta de genotipos tolerantes a enfermedades y altamente productivos, que permitan satisfacer la demanda interna y abrir paso a los mercados internacionales (Sperizen, 2004; Agronet, 2008). En este sentido, pocos son los esfuerzos realizados en el caribe colombiano para explotar la variabilidad y desarrollar cultivares para nuestras condiciones ambientales.

Colombia necesita diversificar las áreas agrícolas y elevar los rendimientos y, con ello, la participación en los niveles de exportación de frutas frescas y procesadas, pues nuestra intervención en el mercado internacional es muy baja, a pesar del potencial de producir frutas durante todo el año, debido a factores tecnológicos limitantes, como cultivares no mejorados, baja utilización de irrigación, deficiente manejo y conservación de la calidad de las frutas producidas (Agronet, 2008).

Meletti $\mathcal{E}$ Bruckner (2001) señalan que el mercado de fruta fresca requiere frutos de forma ovalada para una buena clasificación comercial, buena apariencia, resistencia al transporte y con reducidas pérdidas durante el almacenamiento y la comercialización. De igual manera, el sector agroindustrial, prefiere frutas con todas sus cavidades llenas de pulpa, alto contenido de ácidos y sólidos solubles y un color uniforme. El sector industrial en Brasil demanda frutos con porcentaje de pulpa superior al $50 \%$, contenidos de sólidos solubles totales mayor a $15^{\circ}$ Brix, compactos y de color amarillo (Meletti et al. 2000).

El peso del fruto es una característica importante en el mejoramiento genético, ya que está positivamente relacionado con el rendimiento. Los frutos deben ser grandes y de forma ovalada para conseguir una buena clasificación comercial, así mismo, de cáscara fina y cavidades internas llenas que permitan un mayor rendimiento de jugo (Meletti et al. 2000).

La variabilidad genética intra e interespecífica ha sido reportada por muchos investigadores (Cunha, 1996; Fajardo et al. 1998; Crochemore et al. 2003; Viana et al. 2003; Ganga et al. 2004; De Moraes et al. 2005), lo que señala la potencialidad de mejorar sustancialmente los rendimientos y la calidad de fruto.

Para entender mejor las causas entre las asociaciones entre caracteres, Wright (1921) propuso el análisis de sendero que desdobla las correlaciones estimadas en efectos directos e indirectos de caracteres sobre una variable básica. Los análisis han sido aplicados, en varias especies hortofrutícolas, como en pimentón Capsicum annuum L (De Carvalho et al. 1999) y berenjena Solanum melongena L. (Ingale \& Patil, 1995). 
Falconer E Mackay (1996) y Cruz E Regazzi (1997) señalan que los coeficientes de correlación, a pesar de ser de gran utilidad en la cuantificación de la magnitud y dirección de las influencias de factores en la determinación de caracteres complejos, no dan una exacta importancia relativa de los efectos directos e indirectos de esos factores; la solución a ese problema, se logra con el análisis de sendero.

El análisis de sendero consiste en desdoblar el coeficiente de correlación (fenotípico, genético o ambiental), en los efectos directos e indirectos de varios caracteres (causas), sobre una variable básica compleja (efecto). A pesar que una correlación es una característica intrínseca a dos caracteres en una condición experimental dada, su descomposición es dependiente del conjunto de caracteres estudiados, las cuales, normalmente, son evaluadas por el conocimiento previo del investigador, con base en su importancia y las posibles interrelaciones expresadas anteriormente (Falconer \& Mackay, 1996; Cruz $\&$ Regazzi, 1997; Vencovsky $\&$ Barriga, 1992).

El estimador dentro del análisis de sendero que mide las relaciones causa - efecto entre caracteres es llamado coeficiente de sendero (path coefficient), el cual, mide la influencia directa de una variable sobre otra, independientemente de las demás. Es este estadístico que permite desdoblar los coeficientes de correlación simples en sus efectos directos e indirectos.

Un análisis de sendero es, en esencia, un estudio que permite evaluar si una relación entre dos variables es de causa - efecto o es determinada por la influencia de otra u otras variables. Por lo tanto, un análisis de sendero puede ser realizado a partir de correlaciones fenotípicas, genéticas o ambientales (Cruz E Regazzi, 1997; Vencovsky \& Barriga, 1992). La descomposición de las correlaciones (en efectos directos e indirectos) entre los caracteres agronómicos más importantes es de fundamental importancia, para el logro eficiente de los objetivos en los programas de mejoramiento genético de esta especie.

El análisis de sendero ha sido utilizado con mucho éxito a través del tiempo, como herramienta de gran ayuda en el proceso de selección de plantas y de animales. Diversos estudios sobre correlaciones entre rendimiento y sus componentes, propiedades del fruto, del jugo y de la calidad de la semilla en maracuyá amarillo ( $P$. edulis), han sido reportados por algunos autores (Coelho et al. 2007; Pio et al. 2003; Albuquerque et al. 2002; De Oliveira, 1980; Ferreira et al. 1975), sin embargo, los estudios sobre análisis de sendero para las propiedades del fruto son muy escasos (Negreiros et al. 2007; Coelho et al. 2007).

Estudios realizados en diversas zonas productoras de maracuyá en Brasil, por Albuquerque et al. (2002), por De Oliveira (1980) y por Ferreira et al. (1975), indican una correlación genética alta y positiva $(r>0,67)$ entre el peso del fruto, el diámetro ecuatorial del fruto y el peso de la semillas con arilo (pulpa). Negreiros et al. (2007) trabajando con 200 frutos de un ensayo de 42 progenies de medios hermanos de maracuyá amarillo, encontraron que el análisis de sendero evidenció que el peso de la cáscara y de la pulpa poseen alta correlación con el peso del fruto, donde el peso de la pulpa posee mayor efecto directo. De igual forma, hallaron que el diámetro ecuatorial del fruto presentó mayor efecto directo positivo sobre el rendimiento de pulpa, con relación a la longitud del fruto.

La literatura colombiana sobre mejoramiento genético de maracuyá, no reporta estudios sobre análisis de sendero para las propiedades del fruto, que maximicen la selección y el logro de progresos genéticos en el mejoramiento clásico de la especie, en aras de satisfacer las necesidades de productores y el sector agroindustrial.

El estudio tuvo como objetivos realizar dos análisis de sendero: uno para el peso del fruto en función del peso de la cáscara (pericarpio), peso de la semilla con arilo (pulpa) y peso de la semilla sin arilo (semilla) y, otro, para el peso de la semilla con arilo (pulpa) en función del peso, diámetro ecuatorial, diámetro polar y peso de la semilla sin arilo del fruto.

\section{MATERIALES Y MÉTODOS}

El estudio, se realizó en el año 2006, en la vereda San Isidro (Montería, Córdoba), ubicada en las coordenadas geográficas $8^{\circ} 35^{\prime}$ 09" de latitud norte $(\mathrm{N})$ y $75^{\circ} 53^{\prime}$ 42 " de longitud oeste $(\mathrm{W})$, respecto al meridiano de Greenwich, a 48msnm, humedad relativa promedio de $85 \%$, precipitación media de $1.600 \mathrm{~mm}$ anual, temperatura media de $28^{\circ} \mathrm{C}$. Pertenece a la zona climática calido-moderada, según la clasificación de 
Holdridge, a la formación Bosque Seco Tropical (BST) y a la zona agroecológica Cj (Palencia et al. 2006; IGAC, 1985).

Con la participación activa de productores líderes en toda la zona, se escogieron diez lotes comerciales (Tabla 1) de buena producción y, en cada uno de éstos, se eligieron diez plantas por su vigor, precocidad, sanidad, prolificidad y propiedades comerciales deseables del fruto (amarillos, grandes, elongados y sanos), para un total de 100 plantas. En cada planta, se seleccionaron cuatro frutos por sus características comerciales deseables.

Tabla 1. Coordenadas geográficas de los diez lotes comerciales de maracuyá estudiados.

\begin{tabular}{|c|c|}
\hline Lotes & Coordenadas \\
\hline 1 & $8^{\circ} 35^{\prime} 09^{\prime \prime} \mathrm{N}$ y $75^{\circ} 53^{\prime} 42^{\prime \prime} \mathrm{W}$ \\
\hline 2 & $8^{\circ} 35^{\prime} 05^{\prime \prime} \mathrm{N}$ y $75^{\circ} 53^{\prime} 44^{\prime \prime} \mathrm{W}$ \\
\hline 3 & $8^{\circ} 35^{\prime} 04^{\prime \prime} \mathrm{N}$ y $75^{\circ} 53^{\prime} 40^{\prime \prime} \mathrm{W}$ \\
\hline 4 & $8^{\circ} 35^{\prime} 41^{\prime \prime} \mathrm{N}$ y $75^{\circ} 54^{\prime} 17^{\prime \prime} \mathrm{W}$ \\
\hline 5 & $8^{\circ} 34^{\prime} 54^{\prime \prime} \mathrm{N}$ y $75^{\circ} 54^{\prime} 68^{\prime \prime} \mathrm{W}$ \\
\hline 6 & $8^{\circ} 34^{\prime} 54^{\prime \prime} \mathrm{N}$ y $75^{\circ} 53^{\prime} 34^{\prime \prime} \mathrm{W}$ \\
\hline 7 & $8^{\circ} 34^{\prime} 03^{\prime \prime} \mathrm{N}$ y $75^{\circ} 54^{\prime} 33^{\prime \prime} \mathrm{W}$ \\
\hline 8 & $8^{\circ} 34^{\prime} 07^{\prime \prime} \mathrm{N}$ y $75^{\circ} 54^{\prime} 35^{\prime \prime} \mathrm{W}$ \\
\hline 9 & $8^{\circ} 34^{\prime} 31^{\prime \prime} \mathrm{N}$ y $75^{\circ} 52^{\prime} 53^{\prime \prime} \mathrm{W}$ \\
\hline 10 & $8^{\circ} 34^{\prime} 28^{\prime \prime} \mathrm{N}$ y $75^{\circ} 52^{\prime} 49^{\prime \prime} \mathrm{W}$ \\
\hline
\end{tabular}

Los frutos se llevaron al laboratorio de fitomejoramiento de la Universidad de Córdoba, donde se realizó la estimación de 16 caracteres relacionados con las propiedades físicas y químicas del fruto. Sin embargo, para este estudio, sólo se consideraron por su mayor importancia y nivel de correlación, los caracteres: 1) peso del fruto (PFR), 2) peso de la cáscara o pericarpio (PCA), 3) diámetro ecuatorial (DEC), 4) diámetro polar (DPO), 5) peso de la semilla con arilo o pulpa (PSC) y 6) peso de la semilla sin arilo o semilla (PSA).

La investigación se implementó bajo un diseño experimental anidado con diez lotes de maracuyá, diez plantas dentro de cada lote y cuatro frutos (repeticiones), en cada planta. La estimación de los coeficientes de correlación fenotípicos, genéticos y ambientales, los coeficientes de sendero (efectos directos) y los efectos indirectos, se llevaron a cabo mediante el uso del programa computacional GENES versión Windows (2004.2.1), desarrollado por Cruz (2004).
En total, se realizaron cuatro análisis de sendero: dos para el sistema PFR (variable efecto), en función del PCA, PSC y PSA (variables causas) y, otro para PSC (variable efecto), en función del PFR, DEC, DPO y PSA (variables causas). Los dos análisis de sendero en cada sistema, uno se origina de la realización del estudio con base en el uso de la matriz de correlaciones fenotípicas y, el otro, de la matriz de correlaciones genéticas. Ambas matrices, las origina, automáticamente el programa GENES en el procedimiento para el análisis de varianza (Tabla 2).

Para estimar los efectos directos en cada uno de los análisis, GENES utiliza una matriz de correlaciones (fenotípica o genética: dependiendo el interés), la descompone y la organiza en el siguiente sistema de matrices: $P=A^{-1}$. R; en donde: $A^{-1}$ es la inversa de la matriz de correlaciones (entre cada una de las variables causas), $\mathrm{R}$ es el vector de coeficientes de correlaciones entre las variables causas con la variable efecto y $\mathrm{P}$ es vector de coeficientes de sendero. La descomposición 
Tabla 2. Correlaciones fenotípicas $(\mathrm{rF})$, genéticas ( $\mathrm{rG}$ ) y ambientales $(\mathrm{rE})$ entre seis caracteres asociados al fruto.

\begin{tabular}{|c|c|c|c|c|c|c|}
\hline VARIABLES ${ }^{1}$ & $r$ & DEC & DPO & PCA & PSC & PSA \\
\hline PFR & $\begin{array}{l}r_{F} \\
r_{G} \\
r_{E}\end{array}$ & $\begin{array}{l}0,88^{* *} \\
0,89^{* *} \\
0,85^{* *}\end{array}$ & $\begin{array}{c}0,76^{\star *} \\
0,78^{\star *} \\
0,70^{*}\end{array}$ & $\begin{array}{l}0,95^{\star *} \\
0,97^{* *} \\
0,88^{* *}\end{array}$ & $\begin{array}{l}0,87^{* *} \\
0,92^{\star *} \\
0,87^{* *}\end{array}$ & $\begin{array}{l}0,79^{\star *} \\
0,78^{*} \\
0,86^{* *}\end{array}$ \\
\hline DEC & $\begin{array}{l}r_{F} \\
r_{G} \\
r_{E}\end{array}$ & & $\begin{array}{c}0,74^{* *} \\
0,77^{* *} \\
0,67^{*}\end{array}$ & $\begin{array}{l}0,87^{* *} \\
0,89^{* *} \\
0,81^{* *}\end{array}$ & $\begin{array}{c}0,71^{*} \\
0,77^{* *} \\
0,69^{*}\end{array}$ & $\begin{array}{l}0,61^{*} \\
0,60^{*} \\
0,70^{*}\end{array}$ \\
\hline DPO & $\begin{array}{l}r_{F} \\
r_{G} \\
r_{E}\end{array}$ & & & $\begin{array}{c}0,75^{\star *} \\
0,78^{\star *} \\
0,63^{*}\end{array}$ & $\begin{array}{l}0,61^{*} \\
0,68^{*} \\
0,59^{*}\end{array}$ & $\begin{array}{l}0,57^{*} \\
0,59^{*} \\
0,63^{*}\end{array}$ \\
\hline PCA & $\begin{array}{l}r_{F} \\
r_{G} \\
r_{E}\end{array}$ & & & & $\begin{array}{c}0,67^{*} \\
0,80^{\star *} \\
0,56^{*}\end{array}$ & $\begin{array}{c}0,67^{*} \\
0,74^{\star *} \\
0,65^{*}\end{array}$ \\
\hline PSC & $\begin{array}{l}r_{F} \\
r_{G} \\
r_{E}\end{array}$ & & & & & $\begin{array}{l}0,82^{\star *} \\
0,81^{* *} \\
0,82^{* *}\end{array}$ \\
\hline
\end{tabular}

${ }^{*},{ }^{* *}$ significativos al $5 \%$ y $1 \%$ de probabilidad, respectivamente.

1. Peso del fruto (PFR), diámetro ecuatorial (DEC), diámetro polar (DPO), peso de la cáscara (PCA), peso de la semilla con arilo (PSC) y peso de la semilla sin arilo (PSA).

de los coeficientes de correlación de cada una de las variables causa con la variable efecto $\left(\mathrm{r}_{\mathrm{xi}}\right)$, en sus componentes efecto directo $(\mathrm{Pi})$ y efecto indirecto (Ei), permite, mediante el despeje de las siguientes ecuaciones, estimar los respectivos efectos indirectos (Ei) de cada variable causa:

$$
\begin{array}{ll}
r_{X 1 Y}=P_{1}+E_{1} & \text { : para con PCA } \\
r_{X 2 Y}=P_{2}+E_{2} & \text { : para con PSC } \\
r_{X 3 Y}=P_{3}+E_{3} & \text { : para con PSA }
\end{array}
$$

El coeficiente de sendero, debido a los efectos residuales o a otras variables no consideradas en el estudio (h), la estima mediante la siguiente ecuación:

$$
h=\left[1-\left(P_{1} \cdot r_{X 1 Y}\right)-\left(P 2 \cdot r_{X 2 Y}\right)-\left(P 3 \cdot r_{X 3 Y}\right)\right]^{1 / 2} .
$$

De forma similar, se puede extender el modelo cuando el sistema considera más de tres variables causas, como es el caso de PSC (variable efecto), en función del PFR, DEC, DPO y PSA (variables causas).

\section{RESULTADOS Y DISCUSIÓN}

Las magnitudes y los sentidos de los efectos directos e indirectos derivados de la descomposición de las correlaciones fenotípicas y genéticas para el PFR en función del PCA, PSC y PSA, se presentan en la tabla 3. Se observa que el coeficiente de determinación $\left(R^{2}\right)$ en el análisis de sendero fenotípico y genético para el PFR estuvo por encima del 99\%, es decir, que el 99\% de la variabilidad del PFR fue explicada por las variables: PCA, PSC y PSA, lo cual, indica el buen ajuste del modelo a los datos y la importancia de las tres variables explicativas en la definición del PFR.

Además, se presentó una similitud en la descomposición de las correlaciones en sus efectos directos (en la diagonal) e indirectos (por fuera de la diagonal), para cada una de las variables, entre el análisis con base a las correlaciones fenotípicas y genéticas tanto en sentido, como en magnitud, lo que indica que la relación entre el PFR y las variables explicativas se debe a factores genéticos y no a la existencia de efectos ambientales. 
Tabla 3. Análisis de sendero para el peso del fruto (PFR) en función del peso de la cáscara (PCA), peso de la semilla con arilo (PSC) y peso de la semilla sin arilo (PSA).

\begin{tabular}{|c|c|c|c|c|}
\hline \multirow{2}{*}{ VARIABLES } & \multicolumn{3}{|c|}{ CORRELACIONES FENOTÍPICAS } & \multirow{2}{*}{$\begin{array}{c}r_{F} \\
\text { con PFR }\end{array}$} \\
\hline & PCA & PSC & PSA & \\
\hline PCA & 0,66 & 0,29 & $-0,04$ & $0,95^{\star *}$ \\
\hline PSC & 0,44 & 0,43 & $-0,05$ & $0,87^{* *}$ \\
\hline \multirow[t]{4}{*}{ PSA } & 0,44 & 0,34 & $-0,01$ & $0,79^{* *}$ \\
\hline & \multicolumn{3}{|c|}{$R^{2}=0,995$} & \\
\hline & \multicolumn{3}{|c|}{ CORRELACIONES GENÉTICAS } & $r_{G}$ \\
\hline & PCA & PSC & PSA & con PFR \\
\hline PCA & 0,68 & 0,35 & $-0,05$ & $0,97^{\star *}$ \\
\hline PSC & 0,54 & 0,43 & $-0,06$ & $0,92^{* *}$ \\
\hline \multirow[t]{2}{*}{ PSA } & 0,50 & 0,35 & $-0,07$ & $0,78^{* *}$ \\
\hline & \multicolumn{2}{|c|}{$R^{2}=1$} & & \\
\hline
\end{tabular}

${ }^{*},{ }^{* *}$ significativos al $5 \%$ y $1 \%$ de probabilidad, respectivamente.

En ambos análisis, el efecto directo del PCA sobre el PFR es positivo $(0,66$ y 0,68$)$ y explica, principalmente, la asociación significativa entre PCA y PFR. El efecto directo fue mayor que los indirectos, siendo $2,28(0,66$ / 0,29) a 16,50 (0,66 / -0,04) veces en las correlaciones fenotípicas y 1,93 (0,68 / 0,35) a 13,6 (0,68 / -0,05) en las genéticas. Estos resultados concuerdan con los obtenidos por Negreiros et al. (2007), quienes encontraron que el análisis de sendero evidenció que el peso de la cáscara presentó alta correlación con el peso del fruto y su efecto directo fue de 0,5359.

El nivel de correlación tanto fenotípica $\left(\mathrm{r}_{\mathrm{F}}=0,87\right)$ como genética $\left(r_{\mathrm{G}}=0,92\right)$, entre el PSC y el PFR, se explica por la participación de los efectos directo e indirecto, aunque con ligera mayor importancia del efecto indirecto vía peso de la cáscara (PCA), con valores de 0,44 y 0,54, con el uso de la correlación fenotípica y genética, respectivamente. Ello sugiere que el grado de asociación significativa y directa existente entre el PSC y PFR, se debe en mayor proporción a la influencia indirecta del PCA. Por lo tanto, para realizar selección de frutos por mayor peso es necesario elegir frutos con mayor PCA y PSC.
La descomposición de las correlaciones $\left(\mathrm{r}_{\mathrm{F}}=0,79\right.$ y $\left.\mathrm{r}_{\mathrm{G}}=0,78\right)$ entre el PSA y PFR, está explicada solamente por los efectos indirectos vía PCA y PSC, siendo superior el efecto indirecto, a través del PCA, con valores de 0,44 y 0,50 en el análisis fenotípico y genético, respectivamente. Esto indica que la correlación significativa y directa existente entre el PSA y PFR, se debe en mayor proporción a la influencia indirecta, a través de PCA y PSC.

Con base en este primer análisis de sendero, se puede inferir que, la selección de frutos con mayor peso de cáscara (PCA) permite la obtención directa de frutos más pesados y, de forma indirecta, con mayor peso de semillas con arilo (pulpa) y semillas sin arilo (semillas).

En el análisis de sendero para el PSC en función del PFR, DEC, DPO y PSA se presenta en la tabla 4 y se observa que el coeficiente de determinación $\left(R^{2}\right)$ en el análisis de sendero fenotípico y genético estuvo por encima del $80 \%$, es decir, que el $80 \%$ de la variabilidad del PSC es explicada por las variables PFR, DEC, DPO y PSA, lo cual, indica el buen ajuste del modelo a los datos y la importancia de las variables explicativas en la 
definición del PSC. No obstante, la suma de los efectos directos e indirectos no originan el valor exacto de la correlación fenotípica esperado para las variables causas (PFR, DEC, DPO y PSA) con la variable efecto (PSC), debido a la presencia de importantes efectos residuales
( $h=0,43$ y 0,35 ) originados por la influencia de otras variables no incluidas en el análisis de sendero para el sistema considerado de PSC en este estudio, como por ejemplo: peso y grosor de la cáscara y cavidad del fruto para la pulpa (Negreiros et al. 2007).

Tabla 4. Análisis de sendero para el peso de la semilla con arilo (PSC) en función del peso de fruto (PFR), diámetro ecuatorial (DEC), diámetro polar (DPO) y peso total de la semilla (PSA).

\begin{tabular}{|c|c|c|c|c|c|}
\hline \multirow{2}{*}{ VARIABLES } & \multicolumn{4}{|c|}{ CORRELACIONES FENOTÍPICAS } & \multirow{2}{*}{$\begin{array}{c}r_{F} \\
\text { con PSC }\end{array}$} \\
\hline & PFR & DEC & DPO & PSA & \\
\hline PFR & 0,80 & $-0,11$ & $-0,01$ & 0,25 & $0,87^{* *}$ \\
\hline DEC & 0,70 & $-0,01$ & 0,20 & 0,19 & $0,71^{*}$ \\
\hline DPO & 0,61 & $-0,09$ & $-0,09$ & 0,18 & $0,61^{*}$ \\
\hline \multirow[t]{4}{*}{ PSA } & 0,63 & $-0,07$ & $-0,05$ & 0,32 & $0,82^{* *}$ \\
\hline & \multicolumn{4}{|c|}{$R^{2}=0,81$} & \\
\hline & \multicolumn{4}{|c|}{ CORRELACIONES GENÉTICAS } & $r_{G}$ \\
\hline & PFR & DEC & DPO & PSA & con PSC \\
\hline PFR & 0,93 & $-0,11$ & $-0,07$ & 0,16 & $0,92^{* *}$ \\
\hline DEC & 0,83 & $-0,12$ & $-0,07$ & 0,13 & $0,77^{* *}$ \\
\hline DPO & 0,73 & $-0,09$ & $-0,09$ & 0,12 & $0,68^{*}$ \\
\hline \multirow[t]{2}{*}{ PSA } & 0,73 & $-0,07$ & $-0,05$ & 0,21 & $0,81^{* *}$ \\
\hline & \multicolumn{2}{|c|}{$R^{2}=0,88$} & \multicolumn{2}{|c|}{$\mathrm{h}=0,35$} & \\
\hline
\end{tabular}

${ }^{*},{ }^{* *}$ significativos al $5 \%$ y $1 \%$ de probabilidad, respectivamente.

Los resultados muestran similitud entre el análisis con base en las correlaciones fenotípicas y genéticas, tanto en sentido como en magnitud, lo que indica que la relación entre el peso de la semilla con arilo (PSC) y las variables explicativas obedece a una mayor influencia de efectos genéticos que ambientales. En ambos análisis, el efecto directo del peso del fruto (PFR) sobre el peso de la semilla con arilo o pulpa (PSC) es positivo y es el que explica, principalmente, la asociación significativa entre PSC y PFR, con valores de coeficientes de sendero fenotípico y genético de 0,80 y 0,93 , respectivamente. Este efecto directo fue mayor $3,2(0,80 / 0,25)$ a $80(0,80$ $/-0,01)$ veces en las correlaciones fenotípicas y 5,8 (0,93 / 0,16) a 13,3 (0,93 / -0,07) veces en las correlaciones genéticas, comparados con los efectos indirectos en ambos análisis, en otras palabras, la asociación existente entre el PSC y el PFR no obedece al efecto indirecto del DEC, DPO y PSA. Estos resultados están de acuerdo con los obtenidos por Negreiros et al. (2007), Albuquerque et al. (2002), Oliveira (1980) y Ferreira et al. (1975) quienes han encontrado correlación genética alta y positiva $(r>0,67)$ entre el peso del fruto con el peso de la semillas con arilo (pulpa), señalando que frutos con mayor PFR presentan mayor peso de pulpa (PSC).

La descomposición de las correlaciones ( $r=0,71$ y 0,77 ) entre DEC con PSC, señala que es el efecto indirecto vía PFR $(0,70$ y 0,83$)$ que explica el nivel de asociación significativo entre ellos en ambos análisis. Este efecto indirecto representa 70 y 6,92 veces el efecto directo en el análisis fenotípico y genético, respectivamente. Similar situación, se presenta para el análisis de sendero 
entre DPO con PSC y PSA con PSC, ya que sus efectos indirectos vía PFR son los que explican el nivel de correlación significativo entre ellos en ambos análisis. Los efectos indirectos de DPO, a través de PFR $(0,61$ y $0,73)$, representaron entre 6,80 a 8,11 veces los efectos directos. A su vez, los efectos indirectos de PSA, a través de PFR $(0,63$ y 0,73), indicaron entre 1,97 a 3,50 veces los efectos directos. Lo anterior muestra una posible selección indirecta de la cantidad de pulpa por medio de la selección basada en el PFR.

Con base en los resultados obtenidos en este segundo análisis de sendero, se puede deducir que la selección de frutos con mayor peso (PFR) permite la obtención de frutos con mayor contenido de pulpa (PSC), de manera directa e indirecta (Negreiros et al. 2007). Esto señala que el PFR se puede utilizar eficientemente como criterio para lograr progresos genéticos en la selección de frutos con mayor contenido de pulpa y semilla, mientras que el DEC, DPO, PSA, no son un buen criterio de selección, para lograr el mismo propósito. Sin embargo, ello se puede convertir en una dificultad en el mejoramiento genético, ya que el peso de frutos está asociado con un menor número de frutos por planta (Viana $\mathcal{E}$ Menezes, 2005). El PFR se justifica, además, porque es un carácter más fácil de medir, posibilitando evaluar mayor cantidad de plantas o progenies en el proceso de selección, en comparación, incluso, con el PCA (pericarpio), la cual, mostró también alta correlación genética con PFR ( $\mathrm{rG}$ $\left.=0,97^{*}\right)$, explicada principalmente por sus efectos directos con ésta $(0,68)$.

Los resultados obtenidos son de mucha importancia en los programas de mejoramiento genético de maracuyá, que buscan responder a la demanda de frutos con mayor contenido de pulpa ( $>$ PSC) para el consumo en fresco y procesado por la agroindustria de jugos e igualmente obtener mayor cantidad de semilla por fruto en las hibridaciones artificiales. Ello es de mayor interés si el fitomejorador hace énfasis en la selección de frutos con mayor peso de cáscara, delgada y compacta, lo cual, ofrecerá, de forma indirecta, mayor resistencia al manipuleo, transporte y mayor cavidad potencial para el llenado de pulpa en el fruto (Negreiros et al. 2007).

La selección de frutos ovales, aunque se puede justificar en virtud de exigencias del mercado por mayor rendimiento de pulpa por fruto, en este estudio al igual que en lo encontrado por Negreiros et al. (2007), sugiere que no deberá ser aplicada con el objetivo de aumentar el rendimiento de pulpa, en razón a que el efecto genético indirecto del diámetro ecuatorial $(\mathrm{DEC}=0,83$ ) es mayor que el del diámetro polar $(\mathrm{DPO}=0,73)$, que es un estimador de la longitud del fruto.

\section{CONCLUSIONES}

El peso de la cáscara (pericarpio) es el carácter que tiene mayor efecto directo sobre el peso del fruto y explica, a través de sus efectos indirectos, las correlaciones de otras variables con el peso del fruto.

El peso de la cáscara y en segundo lugar el de la semilla con arilo, se puede usar como criterio de selección, en el mejoramiento genético de P. edulis f. flavicarpa Deg., para la obtención de frutos con gran peso.

El peso del fruto, se puede emplear como criterio de selección en el mejoramiento genético de $P$. edulis $\mathrm{f}$. flavicarpa Deg., para la obtención de frutos con mayor contenido de pulpa y semilla.

\section{BIBLIOGRAFÍA}

AGRONET. 2008. Análisis y Estadísticas. Ministerio de Agricultura y Desarrollo Rural Disponible desde Internet en http://www.agronet.gov.co (con acceso 23/01/08).

ALBUQUERQUE, A.S.; BRUCKNER, C.H.; CRUZ, C.D.; CASALI, V.W.D.; ARAÚJO, R. DA C.; MOREIRA, A.E.; DE SOUZA, J.A. 2002. Possibilidade de seleção indireta para peso do fruto e rendimento em polpa em maracujá (Passiflora edulis Sims). In memorias: Congresso Brasileiro do Fruticultura, III-17., 2002, Belém. Anais Belém: Embrapa. Cd-Room.

COELHO, E.; FIGUEIREDO, R.D.; FERREIRA, R.; PIO, A.V. 2007. Path analysis for physiological traits that influence seed germination of Passiflora edulis $\mathrm{f}$. flavicarpa Deg. Crop Breeding and Applied Biotech. 7:148-154.

CROCHEMORE, M.L.; MOLINARI, H.B.; COLAUTO, N.M. 2003. Caracterização agromorfológica do maracujazeiro (Passiflora spp). Rev. Brás. Frut. 25(1):5-10. 
CRUZ, C.D. 2004. Programa GENES. Versao Windows. Aplicativo Computacional em Genética e Estatística. Editora UFV. Universidade Federal de Viçosa. Disponible desde Internet en www.ufv.br/dbg/genes/genes.htm. (con acceso 16/01/05).

CRUZ, C.D.; REGAZZI, A.J. 1997. Modelos Biométricos aplicados al Mejoramiento Genético. 2a Ed. Editorial UFV. Brasil. 390p.

CUNHA, M.A.P. 1996. Recursos genéticos e modificações em métodos de seleção para produtividade em maracujá. Rev. Brás. Frut. 18:413-423.

DE CARVALHO, C.G.; RODRIGUES, V.; CRUZ, C.; DIAS, V.W. 1999. Analise de trilha sob multicolinearidade em pimentão. Pesquisa Agropec. Brás. 34(4):603-613.

DE MORAES, M.C.; GERALDI, I.O.; MATTA, F.; CARNEIRO, M.C. 2005. Genetic and phenotypic parameter estimates for yield and fruit quality traits from a single wide cross in yellow passion fruit. Hort Science. 40(7):1978-1981.

DE OLIVEIRA, J.C. 1980. Melhoramento genético de Pasiflora edulis f. flavicarpa Deg. Visando ao aumento de produtividade. Tese (Livre-Docência) - Faculdade de Ciências Agrárias e Veterinárias, Universidade Estadual Paulista, Jaboticabal. 133p.

FAJARDO, D.; FERNANDO, A.; GRUM, M.; TOHME, J.; LOBO, M.; ROCA, W.; SÁNCHEZ, I. 1998. Genetic variation analysis of the genus Passiflora L. using RAPD markers. Euphytica. 101(3):341-347.

FALCONER, D.S.; MACKAY, T. 1996. Introducción a la genética cuantitativa. Cuarta edición. 464p.

FERREIRA, F.R.; VALLINI, P.C.; RUGGIERO, C.; LAMSANCHEZ, A. 1975. Correlações fenotípicas entre diversas características do fruto do maracujá amarelo (Passiflora edulis var. flavicarpa). In memorias: Congresso Brasileiro do Fruticultura, III-3., 1975, Rio de Janeiro. Anais. Rio de Janeiro: Universidade Federal Rural do Rio de Janeiro, p.481-489.

GANGA, R.M.; RUGGIERO, C.; DE MACEDO, E.L.; GARCIA, V.; MANTOVANI, M.; CHAGAS, E.A.; WIC-
KERT, E. 2004. Diversidade genética em maracujeiro amarelo utilizando marcadores moleculares fAFLP. Rev. Brás.Frut. 26(3):494-498.

INGALE, B.V.; PATIL, S.J. 1995. Correlations and path analysis in brinjal. Indian J. Hort. 52(1):55-59.

INSTITUTO GEOGRÁFICO AGUSTÍN CODAZZI (IGAC). 1985. Zonificación Agroecológica de Colombia. Memoria explicativa. Litografia IGAC. Bogotá, D. E. 57p.

MELETTI, L.M.M.; BRUCKNER, C.H. 2001. Melhoramento genético. In: C.H. Bruckner, C.H.; Picanço, M.C. (eds). Melhoramento de fruteiras tropicais. Cinco continentes, Porto Alegre, Brazil. p.345-385.

MELETTI, L.M.M.; DOS SANTOS, R.R.; MINAMIS, K. 2000. Melhoramento do maracujazeiro-amarelo: obtenção do cultivar 'composto' IAC-27. Scientia Agricola. 57(3):491-498.

NEGREIROS, J.R.; ÁLVARES, V.D.; BRUCKNER, C.H.; MORGADO, M.A.; CRUZ, C.D. 2007. Relacao entre características físicas e o rendimiento de polpa de maracujá-amarelo. Rev. Bras. Frut. 29(3):546-549.

PALENCIA, G.; MERCADO, T.; COMBATT, E. 2006. Estudio agroclimático del departamento de Córdoba. Ed. Gráficas del Caribe Ltda. Montería. 126p.

PIO A.V.; SANTANA, T.N.; GONZAGA, M.; MAGALHÃES, M.; MARTINEZ, J.F.; DO AMARAL, A.T. 2003. Simple and canonic correlation between agronomical and fruit quality traits in yellow passion fruit (Passiflora edulis f. flavicarpa) populations. Crop Breeding and Applied Biotech. 3(2):133-140.

SCHWENTESIUS, R.R.; GÓMEZ, M.A. 2008. El mercado de maracuyá. Rev. Electrónica Latinoamericana en Desarrollo sustentable. Vol 1: 1-5 Disponible desde Internet en http://vinculado.org/ (con acceso 15/02/08).

SPERIZEN, E. 2004. Estudio "Oportunidades de Negocio” Maracuyá. Asociación Gremial de Exportadores de Productos no Tradicionales - AGEXPRONT. Guatemala, 20p. 
VENCOVSKY, R.; BARRIGA, P. 1992. Genética Biométrica no Fitomelhoramiento. Sociedad Brasileira de Genética. 496p.

VIANA, P.V.; SANTANA, T.N.; GONZAGA, M.; DE SOUZA, M.G.; MARTINEZ, J.F.; AMARAL, A.T. 2003. Diversidade genética entre genotipos comerciais de maracujazeiro amarelo (Passiflora edulis $\mathrm{f}$. flavicarpa) e entre espécies de passiflora nativas determinada por marcadores RAPD. Rev. Brás. Frut. 25(3):489-493.
VIANA. P.A.; MENEZES, G. 2005. Genética quantitativa aplicada ao melhoramento genético do maracujazeiro. In: Maracujá: germoplasma e melhoramento genético. Ed. Gelape Faleiro, F.; Vilela, N.T.; Braga, M.F. Embrapa - Cerrados. p.243-274.

WRIGHT, S. 1921. Correlations and causation. J. Agr. Res. 20:557-585.

Recibido: Agosto 20 de 2008

Aceptado: Octubre 3 de 2008 